\title{
Culture conditions antagonize lineage-promoting signaling in the mouse blastocyst
}

\author{
Tristan Frum ${ }^{\dagger}$ and Amy Ralston \\ Department of Biochemistry, Michigan State University, East Lansing, Michigan, USA \\ Correspondence should be addressed to A Ralston; Email: aralston@msu.edu \\ ${ }^{\dagger}$ (T Frum is now at Department of Internal Medicine, Gastroenterology, University of Michigan Medical School, Ann Arbor, \\ Michigan, USA)
}

\begin{abstract}
The mouse preimplantation embryo is a paradigm for discovery of the molecular principles governing formation of specific cell types during development. In this Point of View Article, we show that conditions commonly used for ex vivo culture of preimplantation development are themselves antagonistic to a pathway that is critical for blastocyst lineage commitment.

Reproduction (2020) 160 V5-V7
\end{abstract}

Knowledge of the mechanisms that drive lineage decisions during mouse preimplantation development can significantly impact fields of stem cell and reproductive biology. Studies of the first 3 days of development have shown that the initially totipotent blastomeres of the mouse embryo adopt one of three cell fates by the blastocyst stage: trophectoderm (future placenta), primitive endoderm (future yolk sac endoderm), or epiblast (future fetus and additional extraembryonic tissues). Notably, the ultimate ratio of these three cell types is largely invariant among embryos, indicating that robust regulatory mechanisms exist. Remarkably, lineage specification is even achieved in incubator-grown preimplantation embryos, raised outside of the maternal environment, suggesting that the most critical regulatory mechanisms are intrinsic to the embryo. However, the possibility that culture conditions influence the embryo's signaling environment cannot be formally excluded.

Major insight into the roles of cell signaling in preimplantation cell fate decisions has been provided by studies of the fibroblast growth factor (FGF) signaling pathway (reviewed in Soszyńska et al. 2019). Culturing embryos in FGF4 and heparin (referred to as FGF4, hereafter) causes blastomeres to adopt primitive endoderm, over epiblast, fate (Yamanaka et al. 2010). Importantly, the balance between primitive endoderm and epiblast fates is achieved through the exquisite regulation of the specific dose of FGF signaling experienced by the embryo as it develops (Krawchuk et al. 2013).

The ability to culture preimplantation embryos in the presence of small-molecule inhibitors or agonists of signaling has enabled discovery of the gene regulatory networks crucial for early mammalian development. For example, artificially tweaking the level of FGF/ ERK signaling in embryos lacking specific transcription factors (i.e. transcription factor gene knockouts) can reveal whether transcription factors function upstream, downstream, or independently of FGF/ERK signaling. In addition, these assays have provided new insights into the developmental mechanisms in other mammals, including humans (Kuijk et al. 2012, Roode et al. 2012, Boroviak et al. 2015, Piliszek et al. 2017).

Culturing mouse embryos in agonists and antagonists of FGF/ERK signaling is routine; yet, we note that this is typically performed in amino acid-containing KSOM medium containing $1 \mathrm{mg} / \mathrm{mL}$ BSA. This raises the possibility that a bovine serum-derived impurity could alter the embryonic signaling environment. To better understand the embryonic signaling environment, we cultured embryos in the presence of $1 \mathrm{mg} / \mathrm{mL}$ polyvinyl alcohol (PVA), a synthetic macromolecule that can replace BSA in supporting blastocyst development (KSOM without BSA hereafter) (Biggers et al. 1997, Jang et al. 2007) and provide a more defined medium. In embryos cultured for $66 \mathrm{~h}$ starting from the twocell stage (embryonic day E1.5) in KSOM without BSA, we observed quantitatively normal specification of trophectoderm, inner cell mass, epiblast and primitive endoderm cell types (Fig. 1A, B and C). This observation is consistent with prior evidence that PVA supports development of mouse blastocysts in vitro (Biggers et al. 1997).

Having established that KSOM without BSA supports blastocyst lineage specification, we then evaluated 

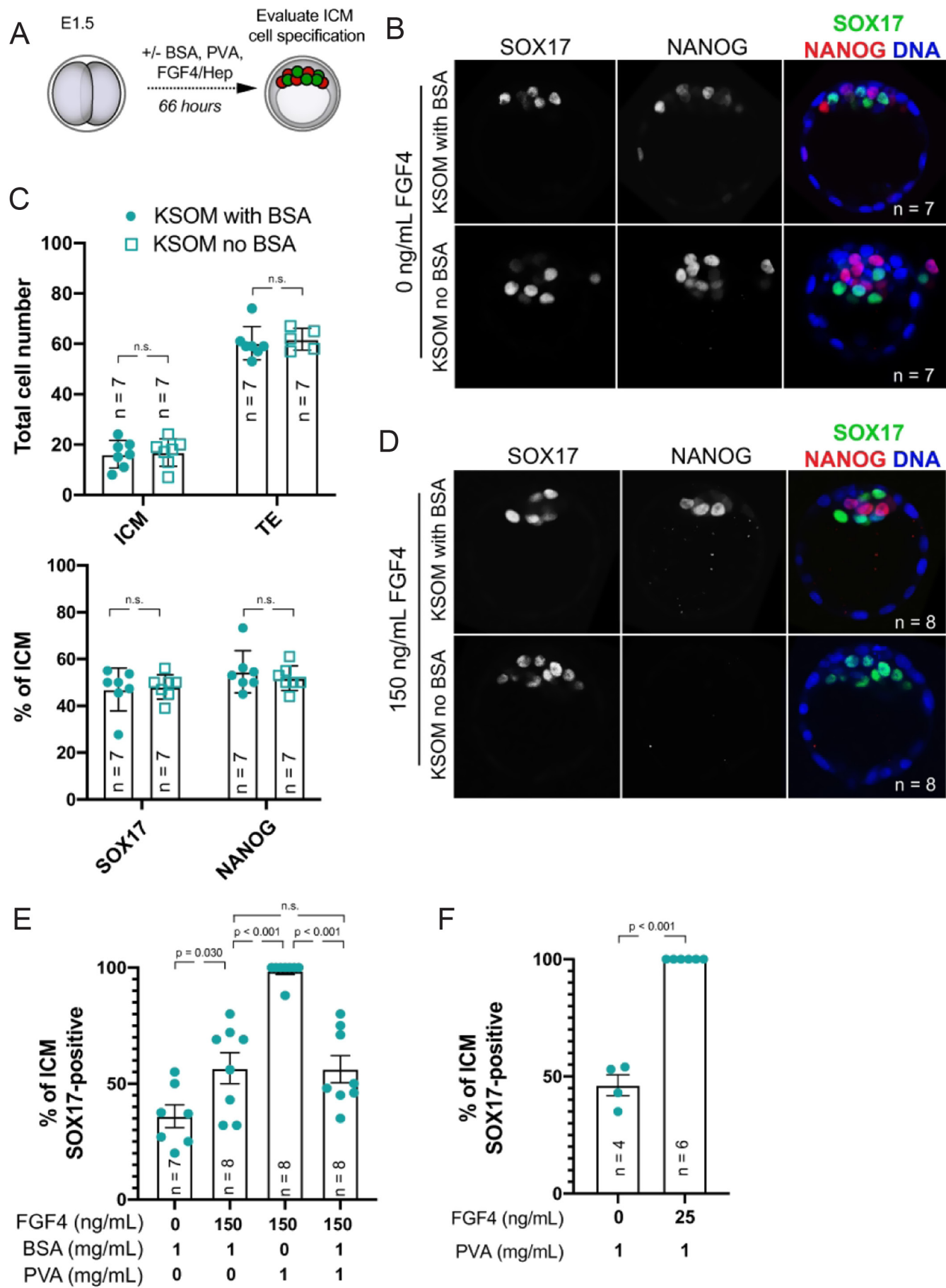

Figure 1 BSA antagonizes signaling by exogenous FGF4. (A) Experimental design for embryos shown and analyzed in this figure. Red=NANOG indicative of epiblast cell fate, green=SOX17 indicative of primitive endoderm cell fate. (B) Immunostaining for SOX17 to identify primitive endoderm (PE) cells and NANOG to identify epiblast (EPI) cells in embryos cultured for 66 hours starting from two-cell stage in KSOM with 1 $\mathrm{mg} / \mathrm{mL}$ BSA or without BSA (Millipore-Sigma MR-121-D and MR-107-D, respectively), but with $1 \mathrm{mg} / \mathrm{mL}$ added PVA (Sigma 360627).

$n=$ number of embryos examined. $P=$ Student's $t$-test. n.s. $=$ not significant $(P>0.2)$. (C) Quantification of the total inner cell mass (ICM) and trophectoderm (TE) cells in embryos cultured in indicated conditions and proportion of ICM cells contributing to PE and EPI in these embryos. Each symbol represents a single embryo, column $=$ mean, and error bars $=$ standard deviations. $P=$ Student's $t$-test. n.s. $=$ not significant $(P>0.2)$. (D) Immunostaining for SOX17 and NANOG in embryos cultured in KSOM+150 ng/mL FGF4 (R\&D Systems 235-F4-025) and $1 \mu \mathrm{mg} / \mathrm{mL}$ heparin (Sigma H3149) in the presence or absence of $1 \mathrm{mg} / \mathrm{mL}$ BSA. Each symbol represents a single embryo, column = mean, and error bars $=$ s.D. $P=$ Student's $t$-test. n.s. $=$ not significant $(P>0.2)$. (E) Quantification of lineage specification in embryos cultured in conditions indicated. Each symbol represents a single embryo, column = mean, and error bars=s.D. (F) Response of embryos cultured in $\mathrm{KSOM}$ without $1 \mathrm{mg} / \mathrm{mL}$ BSA to low dose of exogenous FGF4 and $1 \mu \mathrm{g} / \mathrm{mL}$ heparin. Each symbol represents a single embryo, column $=$ mean, and error bars $=s$.D. $P=S$ tudent's $t$-test. 
the sensitivity of blastocysts to exogenous FGF4 in the absence of BSA. Remarkably, embryos cultured in KSOM without BSA were sensitive to much lower doses of FGF4 $(150 \mathrm{ng} / \mathrm{mL})$ than embryos grown in the presence of BSA (Fig. 1D and E). Higher sensitivity to FGF4 is due to the absence of BSA, rather than the presence of PVA since BSA antagonized the effects of FGF4 signaling in the presence of PVA (Fig. 1E). Surprisingly, a dose as low as $25 \mathrm{ng} / \mathrm{mL}$ FGF4 was capable of driving epiblast cells to express primitive endoderm genes when embryos were cultured in KSOM without BSA (Fig. 1F), which is 20-40 times lower than the FGF4 dose typically used to convert epiblast to primitive endoderm. This observation suggests that higher doses of FGF4 are super-physiological. These observations strongly suggest that BSA is antagonistic to changes in embryonic cell fates induced by exogenous FGF4.

We do not yet understand the mechanism by which BSA interferes with signaling induced by exogenous FGF4. There is precedent that BSA can bind heparin, as well as extracellular signaling pathway members (Francis 2010). We note that BSA does not appear to interfere with endogenous signaling, evidenced by the apparently normal lineage specification occurring in embryos cultured in KSOM with BSA. This could be because BSA in culture cannot penetrate the blastocyst to interfere with the highly localized FGF signaling occurring within the inner cell mass. We therefore caution that the routine supplementation with BSA could complicate studies aimed at understanding the roles and doses of signaling pathways in preimplantation development.

\section{Declaration of interest}

The authors declare that there is no conflict of interest that could be perceived as prejudicing the impartiality of this point of view.

\section{Funding}

This work was supported by the National Institutes of Health award R35 GM131759 to A R.

\section{Author contribution statement}

A R conceived the study and wrote the paper. T F performed experiments, analyzed data, and produced the figure.

\section{Acknowledgements}

Animal use protocols were specifically approved by Michigan State University's Institutional Animal Care and Use Committee. The authors thank Dr Jason Knott for insightful comments and suggestions on the manuscript. They apologize to authors whose work we were unable to cite due to space limitations. They thank the anonymous reviewer for excellent suggestions.

\section{References}

Biggers JD, Summers MC \& McGinnis LK 1997 Polyvinyl alcohol and amino acids as substitutes for bovine serum albumin in culture media for mouse preimplantation embryos. Human Reproduction Update $\mathbf{3}$ 125-135. (https://doi.org/10.1093/humupd/3.2.125)

Boroviak T, Loos R, Lombard P, Okahara J, Behr R, Sasaki E, Nichols J, Smith A \& Bertone P 2015 Lineage-specific profiling delineates the emergence and progression of naive pluripotency in mammalian embryogenesis. Developmental Cell 35 366-382. (https://doi. org/10.1016/j.devcel.2015.10.011)

Francis GL 2010 Albumin and mammalian cell culture: implications for biotechnology applications. Cytotechnology 62 1-16. (https://doi. org/10.1007/s10616-010-9263-3)

Jang M, Lee EJ, Lee ST, Cho M \& Lim JM 2007 Preimplantation and fetal develeopment of mouse embryos cultured in a protein-free, chemically defined medium. Fertility and Sterility 87 445-447. (https://doi. org/10.1016/j.fertnstert.2006.06.021)

Krawchuk D, Honma-Yamanaka N, Anani S \& Yamanaka Y 2013 FGF4 is a limiting factor controlling the proportions of primitive endoderm and epiblast in the ICM of the mouse blastocyst. Developmental Biology 384 65-71. (https://doi.org/10.1016/j.ydbio.2013.09.023)

Kuijk EW, van Tol LT, Van de Velde H, Wubbolts R, Welling M, Geijsen N \& Roelen BA 2012 The roles of FGF and MAP kinase signaling in the segregation of the epiblast and hypoblast cell lineages in bovine and human embryos. Development 139 871-882. (https://doi.org/10.1242/ dev.071688)

Piliszek A, Madeja ZE \& Plusa B 2017 Suppression of ERK signalling abolishes primitive endoderm formation but does not promote pluripotency in rabbit embryo. Development 144 3719-3730. (https:// doi.org/10.1242/dev.156406)

Roode M, Blair K, Snell P, Elder K, Marchant S, Smith A \& Nichols J 2012 Human hypoblast formation is not dependent on FGF signalling. Developmental Biology $361358-363 . \quad$ (https://doi.org/10.1016/j. ydbio.2011.10.030)

Soszyńska A, Klimczewska K \& Suwińska A 2019 FGF/ERK signaling pathway: how it operates in mammalian preimplantation embryos and embryo-derived stem cells. International Journal of Developmental Biology 63 171-186. (https://doi.org/10.1387/ijdb.180408as)

Yamanaka Y, Lanner F \& Rossant J 2010 FGF signal-dependent segregation of primitive endoderm and epiblast in the mouse blastocyst. Development 137 715-724. (https://doi.org/10.1242/dev.043471)

Received 25 February 2020

First decision 10 March 2020

Revised manuscript received 23 April 2020

Accepted 30 April 2020 